\title{
Tuning of a PID Controller for a Real Time Industrial Process using Particle Swarm Optimization
}

\author{
Dr.S.M.Girirajkumar \\ SEEE,SASTRA University \\ Thanjavur,India-613402
}

\author{
Atal.A.Kumar \\ SOME,SASTRA University \\ Thanjavur, India-631402
}

\author{
Dr.N.Anantharaman \\ NIT \\ Tiruchirapalli,India-620015
}

\begin{abstract}
Measurement of level, temperature, pressure and flow parameters are very vital in all process industries. The model for such a real time process is identified and validated . Real time industrial processes are subjected to variation in parameters and parameter perturbations, which when significant makes the system unstable. Determination or tuning of the PID parameters continues to be important as these parameters have a great influence on the stability and performance of the control system. most of the processes are complex and nonlinear in nature resulting into their poor performance when controlled by traditional tuned PID controllers. The need for improved performance of the process has led to the development of optimal controllers. So the control engineers are on look for automatic tuning procedures. This paper discusses in detail about the Particle swarm Optimization (PSO) algorithm, an Evolutionary Computation (EC) technique, and its implementation in PID tuning for an industrial process . Compared to other conventional PID tuning methods, the result shows that better performance can be achieved with the proposed method in terms of time domain specification and performance indices.
\end{abstract}

Keywords: Real time system, PID, automatic tuning, evolutionary computation.

\section{INTRODUCTION:}

Model-based control techniques are usually implemented under the assumption of good understanding of process dynamics and their operational environment. These techniques, however, cannot provide satisfactory results when applied to poorly modelled processes, which can operate in illdefined environments. This is often the case when dealing with complex dynamic systems for which the physical processes are either highly nonlinear or are not fully understood [13]. The conventional proportional-integralderivative (PID) algorithm is still widely used in process industries because its simplicity and robustness. PID controllers are the most common controllers in industry. In fact, $95 \%$ of control loops use PID and the majority is PI control [2]. However, its performance is not adequate in many chemical processes. A change in the signal and the directionality of the process gain is a complex practical situation and, so, still becoming complex the design of a control system [4]. So it becomes necessary to tune the controller parameters to achieve good control performance with the proper choice of tuning constants [2] . Designing and tuning a PID controller demands flexible algorithms, if multiple and conflicting objectives are to be achieved. A conventionally tuned PID controller with fixed parameters may usually derive lesser control performance when it comes to system demands. The conventional tuning techniques lack the intelligence and flexibility which would increase the performance rate and also improvise the stability and error criterion [18, 24].In past few decades, intelligent techniques have been used to meet system demands. An intelligent agent is a system that perceives its environment and takes actions which maximizes its chances of success. Neural network and fuzzy logic mimic the functioning of the human intelligence process [1]. However their real time implementation is quite difficult [23]. On the other hand optimization algorithms have also received increasing attention by the research community as well as the industry [25]. The advantage of optimization algorithms over neural controllers is that they can be incorporated in PID tuning with ease and simplicity. Control design is called "optimal control" when a predefined criterion is optimized [11].Optimality is just with respect to the criterion at hand and the real performance depends on the suitability of the chosen criterion [11].An emergent paradigm is the swarm intelligence [7]. Swarm intelligence approaches present similar population and evolution characteristics to those of evolutionary computation paradigms. However, it differentiates in emphasizing the cooperative behaviour among group members. Swarm intelligence is inspired in nature, in the fact that interactions among a group of living animals contributes with their own experiences to the group, making it stronger in face of others. The most familiar representatives of swarm intelligence in optimization problems are: food-searching behaviour of ants [8], particle swarm optimization [14], bacterial foraging [19], and artificial immune system [5]. A special approach of swarm intelligence based on simplified simulations of animals' social behaviours, such as fish schooling and bird flocking, is the particle swarm optimization (PSO) algorithm $[14,14]$. PSO is a self-adaptive search optimization, first introduced by Kennedy and Eberhart [14]. Once the PSO is based on a simple concept, the social behaviour of particles in the swarm, it has attracted many researchers' attention and has been applied with success to complex engineering problems, mainly in nonlinear function minimization [27], optimal capacitor placement in distribution systems [26], shape optimization [10], dynamic systems and game theory [22], constrained optimization [16], multiobjective optimization problems [3], electromagnetic [22], control systems [21], planning of electrical systems [12], and others Particle swarm optimization (PSO) is a computational algorithm technique based on swarm intelligence. This method is motivated by the observation of social interaction and animal behaviours such as fish schooling and bird flocking. The objective of the paper is to use the PSO algorithm in order to obtain optimal PID controller settings for temperature control in a real time industrial process.

In the forthcoming sections the industrial process considered is explained in detail followed by the conventional tuning technique used, implementation of the algorithm and comparison of the conventional tuning technique with the proposed algorithm in terms of time domain specifications and performance indices are presented.

\section{INDUSTRIAL PROCESS BASED CLOSED LOOP SYSTEM:}

The closed loop system that has been considered here is used to maintain the temperature in an agitated vessel. The agitator 
consists of three paddles which are connected to a vertical shaft. The shaft is connected to an electric motor. The agitator is used to mix two acids namely sulphuric acid $\left(\mathrm{H}_{2} \mathrm{SO}_{4}\right)$ and oleum $\left(\mathrm{H}_{2} \mathrm{~S}_{2} \mathrm{O}_{7}\right)$. There are three inlets, for the inflow of sulphuric acid, oleum and steam. The steam is used to supply heat to the agitating vessel and maintain it at a temperature of $110-130^{\circ} \mathrm{C}$. The steam is supplied from a 10 ton boiler through a regulator which supplies steam at a maximum pressure of about $5 \mathrm{kgf} / \mathrm{cm}^{2}$. The mixed acid solution is then sent to the reactor through an overhead pipeline. The steam from the boiler is sent through a pipeline .This line is connected as inlet to the control valve which controls the steam entering the agitated vessel. A resistance temperature detector (RTD) is used to measure temperature in vessel. The range of RTD used is $0-200^{\circ} \mathrm{C}$. The output signal is conditioned and converted to a current signal, which is of the standard range of $4-20 \mathrm{~mA}$.Here, $4 \mathrm{~mA}$ corresponds to $0^{\circ} \mathrm{C}$ and $20 \mathrm{~mA}$ corresponds to $200^{\circ} \mathrm{C}$ of RTD. The output signal is given to the host computer through the panel board of a Distributed Control System (DCS). Man-Machining Interface allows human interruption in the process whenever necessary through the host computer, which acts as a controller. Then the output signal from the computer is sent to a current to pressure (I/P) converter. The signal is a current signal of 4$20 \mathrm{~mA}$, the $\mathrm{I} / \mathrm{P}$ converter is a device which gives out pressurized air in the range of $3-15$ psi to the control valve proportional to the current supply given to it. The control valve is of globe type which is used to control the steam supplied to the agitated vessel. The steam is supplied through metal pipes of about 2" diameter. When the pressure of the supply air to the control valve from the $\mathrm{I} / \mathrm{P}$ converter is $15 \mathrm{psi}$ the valve will be $100 \%$ opened and when the pressure is $3 \mathrm{psi}$ the valve will be $0 \%$ opened. Thus the valve opening is proportional to the air pressure supplied to it. The steam supplied to the control valve is about $4-5 \mathrm{kgf} / \mathrm{cm}^{2}$, which increases the temperature suitably as per the requirement. The piping and instrument diagram of the process is shown in figure.1.

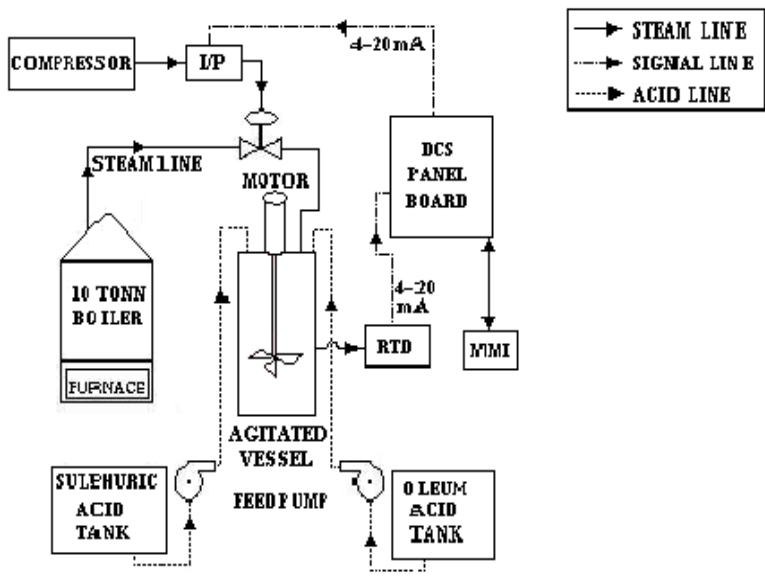

Figure 1.Piping and Instrument diagram of the industrial closed loop process

The industrial process system is further considered as a closed loop system with the components having the specifications as indicated. Mathematical model for this process is estimated by considering a step change of $10 \%$ to the steam valve, after putting the system in an open loop mode. The response curve was traced, and was found similar to be that of a FOPTD, and the mathematical model was found to be,

$$
G(s)=\frac{0.468 e^{-42 s}}{(328 s+1)}
$$

The model validation with its real time response is given in the figure. 2 .

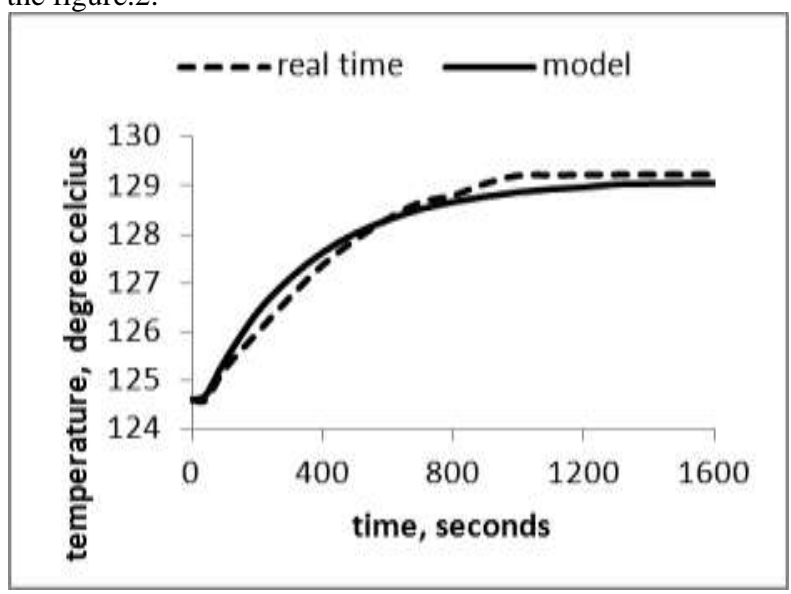

Figure 2. Comparison of real time and model response for the industrial process.

\section{NON-TRADITIONAL OPTIMIZATION TECHNIQUES:}

The implementation of non-traditional optimization techniques for a process based on temperature as the variable to be controlled in a process industry has been attempted. A PID controller is proposed for the system, which fulfills the need for anticipatory control. PID controllers are also considered more suitable for temperature based processes. The transfer function of the process system based on the operating conditions was estimated as in equation 1 .

The conventional method chosen for the proposed work is called Internal Model Control (IMC) technique. The various formulae used for this method for tuning the PID controller are given in table 1 .

Table 1: Tuning rules for IMC technique

\begin{tabular}{|l|c|c|c|}
\hline \begin{tabular}{|l|c|} 
Controll \\
er Type
\end{tabular} & $\begin{array}{c}\text { Controller } \\
\text { Gain (no } \\
\text { units) }\end{array}$ & $\begin{array}{c}\text { Integra } \\
\text { I Time } \\
\text { (second } \\
\text { s) }\end{array}$ & $\begin{array}{c}\text { Derivati } \\
\text { ve Time } \\
\text { (seconds } \\
\text { ) }\end{array}$ \\
\hline $\begin{array}{c}\text { PID } \\
\text { control }\end{array}$ & $\frac{\tau}{K(\lambda+\theta / 2)}$ & $\tau$ & $\theta / 2$ \\
\hline
\end{tabular}

$\theta=$ process dead time (seconds)

$\tau=$ process lag time (seconds)

$\mathrm{K}=$ process gain (dimensionless)

$\lambda=2 \theta$ used for aggressive but less robust tuning

$\lambda=2(\tau+\theta)$ used for more robust tuning

Some controller mechanisms use proportional band instead of gain. Proportional band is equal to 100 divided by gain. The values in the table are for an ideal type controller. The controller computes controller gain, integral time, and derivative time using the formulas shown. 


\section{OPTIMIZATION USING PSO}

The proposal of PSO algorithm was put forward by several scientists who developed computational simulations of the movement of organisms such as flocks of birds and schools of fish. Such simulations were heavily based on manipulating the distances between individuals, i.e., the synchrony of the behavior of the swarm was seen as an effort to keep an optimal distance between them. Sociobiologist Edward Osbourne Wilson outlined a link of these simulations for optimization problems[15]. PSO, originally developed by Kennedy and Eberhart in 1995, is a population-based swarm algorithm[14,9]. In the PSO computational algorithm, population dynamics simulates bio-inspired behaviour, i.e., a "bird flock's behaviour which involves social sharing of information and allows particles to take profit from the discoveries and previous experience of all the other particles during the search for food. Each particle in PSO has a randomized velocity associated to it, which moves through the problem space. Each particle in PSO keeps track of its coordinates in the problem space, which are associated with the best solution (fitness) it has achieved so far. This value is called pbest (personal best). Another "best" value that is tracked by the global version of the particle swarm optimizer is the overall best value. Its location, called gbest (global best), is obtained by any particle in the population. The past best position and the entire best overall position of the group are employed to minimize (or maximize) the solution. The PSO concept consists, in each time step, of changing the velocity (acceleration) of each particle flying toward its pbest and gbest locations (global version of PSO). Acceleration is weighted by random terms, with separate random numbers being generated for acceleration toward pbest and gbest locations, respectively. The concept of PSO is briefly explained in figure. 2 , where $\mathrm{P}^{\mathrm{k}}$ is the current position, $\mathrm{P}^{\mathrm{k}+1}$ is the modified position, $v_{\text {inial }}$ is the initial velocity, $v_{\text {mod }}$ is the modified velocity,$v_{\text {pbest }}$ is velocity considering $\mathrm{p}_{\text {best }}$ and $v_{\text {best }}$ is the velocity considering $\mathrm{g}_{\text {best }}$.

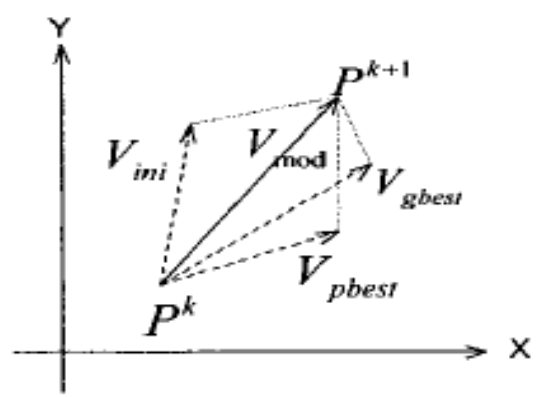

Figure 2. Concept of changing a particle's position in PSO

The fitness function evaluates the performance of particles to determine whether the best fitting solution is achieved. PSO has also been proved successful on diverse engineering applications like logic circuit[14], control design[6,29,17], power system design[20,28] among others.

\section{IMPLEMENTATION OF PSO ALGORITHM:}

The optimal values of the PID controller parameters $K_{p}, K_{i}$ and $\mathrm{K}_{\mathrm{d}}$, is found using PSO. For the PID controller design, it is ensured the controller settings estimated results in a stable closed loop system.

\subsection{Selection of PSO parameters}

To start up with PSO, certain parameters need to be defined. Selection of these parameters decides to a great extent the ability of global minimization.

Population size $=100$

Number of iterations $=100$

Velocity constant, $\mathrm{c} 1=2$

Velocity constant, $c 2=2$

\subsection{Objective Function for the Particle swarm optimization}

The objective functions considered are based on the error criterion. A number of such criteria are available and in this paper controller's performance is evaluated in terms of Integral time absolute error (ITAE) error criteria. The error criterion is given as a measure of performance index given by the equation 2 :

ITAE $=\int_{0}^{T} t|e(t)| d t$

\subsection{Termination Criteria}

Termination of optimization algorithm can take place either when the maximum number of iterations gets over or with the attainment of satisfactory fitness value. Fitness value, in this case is nothing but reciprocal of the magnitude of the objective function, since we consider for a minimization of objective function. In this work the termination criteria is considered to be the attainment of satisfactory fitness value which occurs with the maximum number of iterations as 100 . When the iterations are further increased it is found that there is no significant change in the values obtaibed.

\section{RESULTS AND COMPARISON:}

The implementation of PSO is done to find the optimal PID controller parameters. They are plotted as the best values among the considered population size for all the iterations, and are given in figures 3-5.

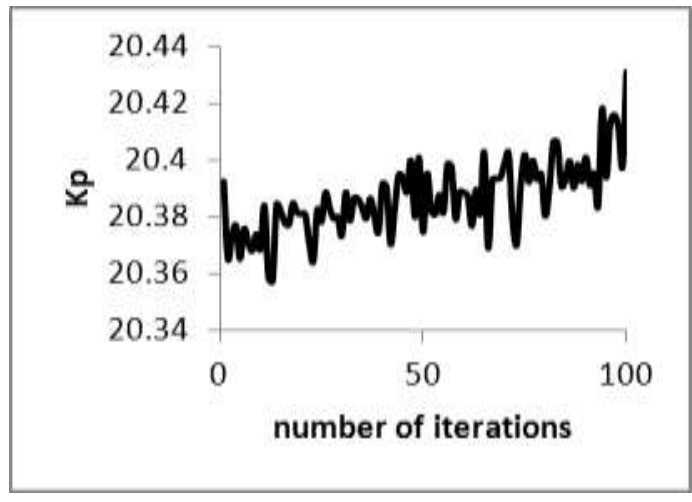

Figure 3. Best solutions of $K_{p}$ for 100 iterations for industrial process based on PSO 


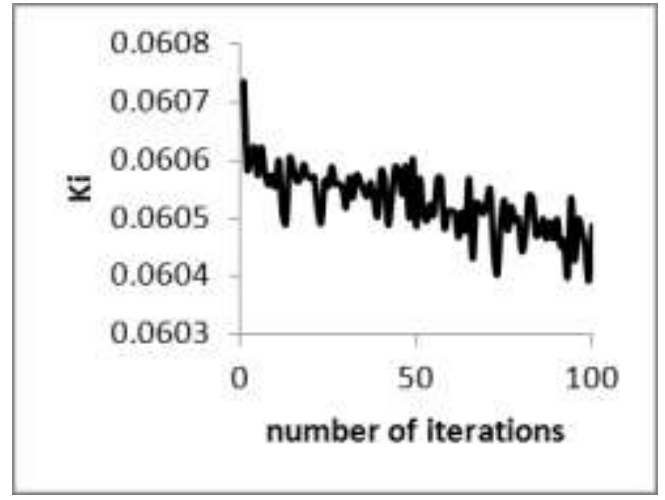

Figure 4. Best solutions of $K_{i}$ for 100 iterations for industrial process based on PSO

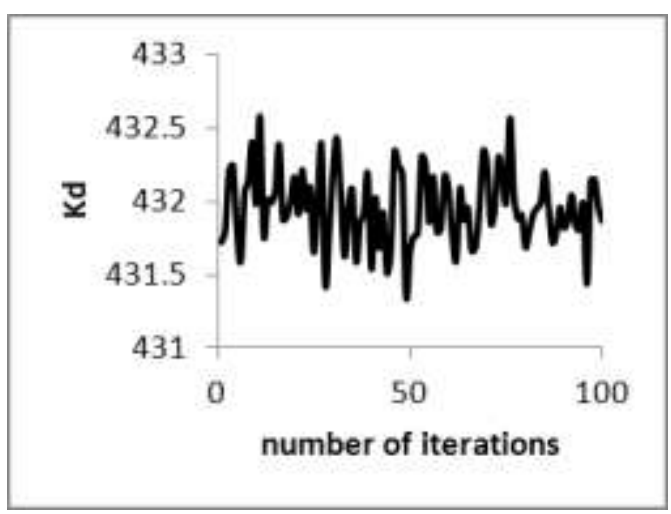

Figure 5. Best solutions of $K_{d}$ for 100 iterations for industrial process based on PSO

The optimal values based on the implementation of PSO for the considered case is given as

\section{$K_{p}=20.431, K_{i}=0.0604$ and $K_{d}=431.86$}

The PID controller is designed for an industrial closed loop process for which the control variable is temperature. The process is allowed to reach the steady state condition at $122^{\circ} \mathrm{C}$ , and the PID controller is studied for its response by giving a servo change in the control variable by $2^{\circ} \mathrm{C}$, making the new set point to be $124^{\circ} \mathrm{C}$. The IMC controller is the best among the traditional techniques. Also, the various PID controller parameters considered for analysis in this section are shown in the below Table 2

Table 2: Various PID controller parameters for industrial process

\begin{tabular}{|c|c|c|}
\hline Controllers & IMC & PSO \\
\hline Proportional gain, $\mathrm{K}_{\mathrm{p}}$ & 3.620 & 20.431 \\
\hline Integral gain constant, $\mathrm{K}_{\mathrm{i}}$ & 0.0103 & 0.0604 \\
\hline Derivative gain constant, $\mathrm{K}_{\mathrm{d}}$ & 71.445 & 431.86 \\
\hline
\end{tabular}

The response of the controlled variable was sketched for the proposed PID controller, and is presented in figure 6 .

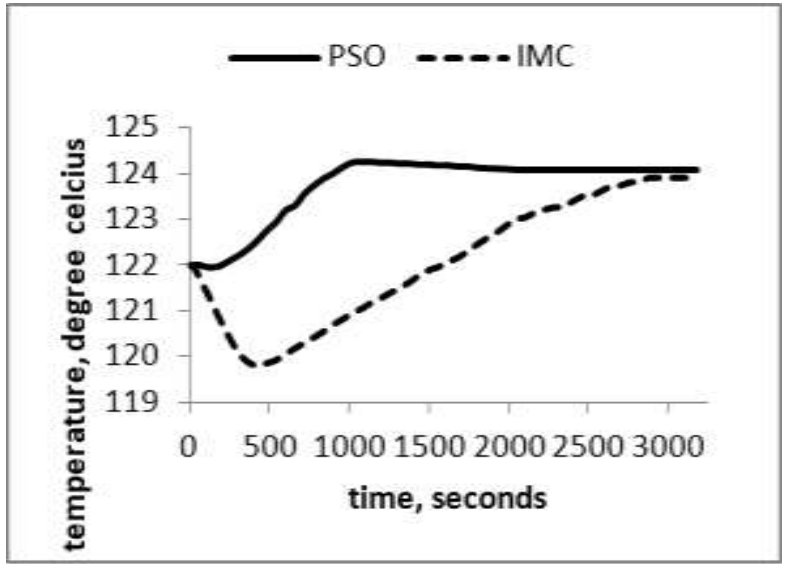

Figure 6. Comparative response of IMC, PSO based controllers for industrial process

Based on these responses, the time domain specifications with relevance to the real time data, is noted and they are tabulated and presented in the Table 3.

Table 3: Time domain specifications for industrial process

\begin{tabular}{|c|c|c|}
\hline \multicolumn{2}{|c|}{ system } & PSO \\
\hline $\begin{array}{c}\text { Inverse peak } \\
\text { (degree) }\end{array}$ & 119.8 & 121.95 \\
\hline $\begin{array}{c}\text { Inverse peak } \\
\text { time(seconds) }\end{array}$ & 360 & 120 \\
\hline $\begin{array}{c}\text { Rise time } \\
\text { (seconds) }\end{array}$ & 2500 & 900 \\
\hline $\begin{array}{c}\text { Peak time } \\
\text { (seconds) }\end{array}$ & 2500 & 1020 \\
\hline $\begin{array}{c}\text { Overshoot } \\
\text { (\%) }\end{array}$ & 1.2 & 13.0 \\
\hline $\begin{array}{c}\text { Settling time } \\
\text { (seconds) }\end{array}$ & 2500 & 1980 \\
\hline
\end{tabular}

The robustness investigation for the process is analyzed by calculating the performance index to the transfer function model whose parameters are deviated by $\pm 20 \%$. The altered model which possesses the uncertainties is given by,

$$
G(s)=\frac{0.561 e^{-33.6 s}}{(393.6 s+1)}
$$

The graph showing the variation of objective function as the iterations are carried on is shown below 


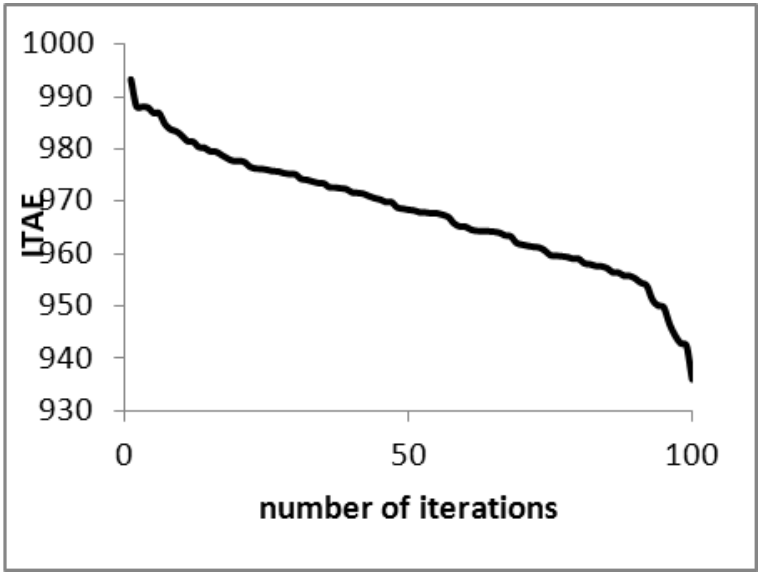

Figure 7. ITAE values for 100 iterations for industrial process based on PSO

The calculation of the performance index for the mentioned model with the proposed controllers are tabulated and presented in the Table 4.

Table 4: Performance index for industrial process model

\begin{tabular}{|l|c|c|}
\hline & IMC & PSO \\
\hline ITAE & 999.03 & 87.03 \\
\hline IAE & 948.11 & 199.86 \\
\hline ISE & 952.63 & 318.23 \\
\hline MSE & 0.0418 & 0.0140 \\
\hline
\end{tabular}

The response curve with the IMC controller has a larger negative peak as the delay is not properly taken care, whereas the PSO controller is the best, proving to be a better one to achieve the set point. It is clear from the responses that the PSO based controller has the advantage of a better closed loop time constant, which enables the controller to act faster with a balanced overshoot and settling time. The response of IMC controller is more sluggish than the PSO based controller. Also, the robustness investigation illustrates the proposed tuning techniques always have a lesser value than the traditional PID controller.

\section{CONCLUSION:}

The various results presented prove the betterness of the PSO tuned PID settings than the IMC tuned ones. The simulation responses for the models validated reflect the effectiveness of the PSO based controller in terms of time domain specifications. The performance index under the various error criterions for the proposed controller is always less than the IMC tuned controller. Above all the real time responses confirms the validity of the proposed PSO based tuning for the industrial process considered.

PSO presents multiple advantages to a designer by operating with a reduced number of design methods to establish the type of the controller, giving a possibility of configuring the dynamic behavior of the control system with ease, starting the design with a reduced amount of information about the controller (type and allowable range of the parameters), but keeping sight of the behavior of the control system. These features are illustrated in this work by considering the problem of designing a control system for a plant of first-order system with time delay and deriving the possible results.

\section{REFERENCES:}

[1] Asriel U. Levin and Kumpati S. Narendra, Control of nonlinear dynamical systems using Neural NetworksPart II : observability, identification and control, IEEE Transactions on Neural Networks, Vol. 7, No. 1, January 1996.

[2] Åström. K. J and Hägglund. T, PID controllers: theory, design, and tuning. Instrument Society of America, ISA, 1995.

[3] Baumgartner U, Magele Ch, Renhart W. Pareto optimality and particle swarm optimization. IEEE Trans Magn;40(2):1172-5,2004.

[4] Bisowarno. B. H, Tian. Y. C, and Tade .M. O, "Model gain scheduling control of an ethyl tert-butyl ether reactive distillation column," Ind. Eng. Chem. Res., vol. 42, pp. 3584-3391, 2003.

[5] Castro LN, Timmis JI. Artificial immune systems: a new computationalintelligence approach. London, UK: Springer-Verlag; 2002.

[6] CoelloC, LunaE. Use of particle swarm optimization to design Combinational logic circuits. In:TyrellA, HaddowP, TorresenJ, editors. $5^{\text {th }}$ International conference on evolvable systems: from biology to hardware, ICES 2003. Lecture notes in computer science, vol.2606. Trondheim, Norway: Springer; p.398-409, 2003.

[7] Coelho.L.D.S, Sierakowski. C. A," A software tool for teaching of particle swarm optimization fundamentals ", Advances in Engineering Software 39 (2008) 877-887

[8] Dorigo M, Di Caro G. The ant colony optimization metaheuristic. In:Corne D, Dorigo M, Glover F, editors. New ideas in optimization. McGraw-Hill; p. 11-32, 1999.

[9] Eberhart. R. C and Kennedy. J. F, "A new optimizer using particle swarm theory," Proceedings of International Symposium on Micro Machine and Human Science, Japan, pp. 39-43, 1995.

[10] Fourie PC, Groenwold AA. The particle swarm optimization algorithm in size and shape optimization. Structural Multidisciplinary Optimization;23(4):25967,2002 .

[11] Javed Alam Jan, Bohumil Sulc, Evolutionary computing methods for optimizing virtual reality process models, International Carpathian control conference ICCC'2002, Malenovice, Czech Republic, May 27-30, 2002.

[12] Kannan S, Slochanal SMR, Subbaraj P, Padhy NP. Application of particle swarm optimization technique and its variant to generation expansion planning problem. Electr Power Sys Res;70(3): 203-10,2004.

[13] Karray .F, Gueaieb. W, and Al-Sharhan .S, "The hierarchical expert tuning of pid controllers using tools of soft computing," IEEE Transactions on Systems, Man, and Cybernetics $\square$ Part B: Cybernetics, vol. 32, no. 1, pp. 77-90, 2002. 
[14] Kennedy JF, Eberhart RC. Particle swarm optimization. In: Proceedingsof the IEEE international conference on neural networks, vol. 4. Perth, Australia; p. 194248,1995 .

[15] Kennedy JF, Eberhart RC, Shi Y. Swarm intelligence. San Francisco: Morgan Kaufman; 2001.

[16] Krohling RA, Coelho LS. Coevolutionary particle swarm optimization using gaussian distribution for solving constrained optimization problems. IEEE Trans Syst, Man and Cybern, Part B: Cybern ;36(6):1407-16,2006.

[17]Krohling R, CoelhoL, ShiY. Cooperative particle swarm optimization for robust control system design.In: $7^{\text {th }}$ Online world conference on soft computing in industrial applications, 2002

[18] Mehrdad Salami and Greg Cain, An adaptive PID controller based on Genetic algorithm processor, Genetic algorithms in engineering systems: Innovations and applications, 1214, September 1995, Conference publication No. 414, IEE 1995.

[19] Muller SD, Marchetto J, Airaghi S, Koumoutsakos P. Optimization based on bacterial chemotaxis. IEEE Trans Evolut Comput ;pp6-29,2002.

[20]Oliveira,P ,CunhaJ, CoelhoJ .Design of pid controllers using the Particle swarm algorithm. In: Twenty-first IASTED international Conference :modelling, identification, and control(MIC2002),Innsbruck,Austria,2002.

[21] Parsopoulos KE, Vrahatis MV. On the computation of all minimizers through particle swarm optimization. IEEE Trans Evolutionary Computaion;8(3):211-24,2004.

[22] Robinson J, Samii YR. Particle swarm optimization in electromagnetic IEEE Trans Antenn Propag ;52(2):397407,2004 .
[23] Simon Fabri and Visakan Kadirkamanathan, Dynamic structure neural networks for stable adaptive control of nonlinear systems, IEEE Transactions on Neural Networks, Vol. 7, No. 5, September1996.

[24] Su Whan Sung, In-Beum Lee and Jitae Lee, Modified Proportional-Integral Derivative (PID) Controller and a New Tuning Method for the PID Controller, Ind. Eng. Chem. Res., 34, pp. 4127-4132, 1995.

[25] Wah. B , Chen. Y, Constrained genetic algorithms and their applications in nonlinear constrained optimization, In Proceedings of International conference on tools with artificial intelligence, IEEE, November 2000, pp. 286293

[26] Yu XM, Xiong XY, Wu YW. A PSO-based approach to optimal capacitor placement with harmonic distortion consideration. Electr Power Sys Res ;vol71(1).pp:27-33, 2004.

[27] Zhang L, Yu H, Hu S. A new approach to improve particle swarm optimization, GECCO 2003. In: Cantu'Paz E. et al., editor. LNCS 2723. Springer, London, UK; p. 1341-39, 2003.

[28] Zhang W, Liu,M. ClercY. An adaptive pso algorithm for reactive Power optimization .In: Sixth international conference on advances inPowersystem control, operation and management (APSCOM) 2003,HongKong,China,p.302-7,2003.

[29]Zheng Y,MaL,ZhangL,QianJ.Robust pid controller design using Particle swarm optimizer.In: IEEE international symposium on Intelligence control,p.974,2003. 